\title{
Philosophiques
}

\section{Chonique de la vie philosophique}

Volume 1, numéro 2, octobre 1974

URI : https://id.erudit.org/iderudit/203019ar

DOI : https://doi.org/10.7202/203019ar

Aller au sommaire du numéro

Éditeur(s)

Société de philosophie du Québec

ISSN

0316-2923 (imprimé)

1492-1391 (numérique)

Découvrir la revue

Citer ce document

(1974). Chonique de la vie philosophique. Philosophiques, 1(2), 157-177.

https://doi.org/10.7202/203019ar d'utilisation que vous pouvez consulter en ligne.

https://apropos.erudit.org/fr/usagers/politique-dutilisation/ 


\section{CHRONIQUE}

\section{de la vie philosophique}

\section{CONGRÈS}

Le 42e Congrès de l'Association Canadienne-française pour l'avancement des sciences (ACFAS) a eu lieu à l'Université Laval du 8 au 10 mai 1974. A cette occasion la section «philosophie » de l'Association a présenté les communications suivantes : J. Danek (Laval), Le Thème de l'intersubjectivité et l'eidos de notre temps, A. Hamel (Laval), Analyse critique de l'opposition intériorité-extériorité dans la phénoménologie de Merleau-Ponty, $\mathrm{V}$. Jabbour (Moncton), L'Univers esthétique dans la phénoménologie de M. Merleau-Ponty, R. Nadeau (UQAM), L'articulation d'un paradigme dans l'épistémologie historique de Thomas $S$. Kubn, G. Leroux (UQAM), La lexicographie philosopbique, F. Duchesneau (Ottawa), Classification et essences nominales selon l'épistémologie de Locke, M. Bunge (McGill), Dialectique et logique, V. Leblanc (Moncton), La relation absolue dans la logique bégélienne, Th. F. Geraets (Ottawa), Les trois syllogismes de la philosophie ou les trois lectures philosophiques de Hegel, E. Joos (Loyola), Les chemins qui ne mènent nulle part, C. Lévesque (Montréal), Position de l'Eternel Retour dans Zaratboustra, J. Philippoussis (Dawson College), Le mythe et l'bomme, A. M. De Rezendo (Trois-Rivières), Les positions de départ dans l'itinéraire de Merleau-Ponty, P. Gravel (Montréal), Hume et la Politique, Différence et Distribution, P. A. Quintin (Trois-Rivières), Signification et valeur des a priori chez Kant, Husserl et les Structuralistes, R. Lebeuf (Montréal), En lisant Parménide, R. Hébert (CEGEP Maisonneuve), L'outil-réflexion: un chapitre dans l'bistoire du concept de réflexion, L. Marcil-Lacoste (McGill), Le professeur de Voltaire et les Canadiens, J. P. Legaré, Pierre Boucher (1622-1717): premier québécois véritablement 
et naturellement philosophe, W. Ross (Montréal), Possibilités et bornes de la pbilosopbie bispano-américaine, L.-M. Vacher (CEGEP d'Ahuntsic), Remarques stratégiques à propos des discours sur l'art : sémiologie, bistoire, critique, B. Rioux (Montréal), Critique de l'ontologie beideggerienne par Lévinas, P. McCormick (Ottawa), Le dernier beidegger et le langage, P. Gagné (TroisRivières), Le problème de la contradiction entre le nationalisme et la lutte des classes, C. Savary et M. Chabot (Trois-Rivières), A propos d'Edmond de Nevers, J. Plamondon, Le statut épistémologique de la praxis chez Marx, M. Lagueux (Montréal), L'inutile insistance sur le rapport des instances, C. Murin (Montréal), Le problème de la périodisation de l'oeuvre et de la vie de Nietzsche, C. Lagadec (Montréal), Eloge de l'obéissance, G. Potvin (Montréal), Quelques problèmes relatifs à la traduction du vocabulaire aristotélicien de la causalité, J. King-Farlow et P. Jones (Alberta), Deux traditions de l'Epistémologie: dialogue sceptique, K. Arnold (Ottawa), La Théorie dite "identité » de l'Esprit, ou l'Hérésie d'Australasie, S. Morin (Moncton), Je me souviens: critique des théories de Shoemaker, Parfit, Pucetti et al. sur l'identité personnelle, G. Helal (Montréal), La nature et la fin de la religion selon $A$. N. Whitebead, M. Renault (Trois-Rivières), De la croyance précritique à l'option personnelle.

En plus de ces communications deux colloques eurent lieu. Le premier portait sur la Méthodologie des Sciences Humaines avec comme participants: V. Cauchy (Montréal), président et C. Savary (Trois-Rivières), Y. Gauthier (Montréal), C. Limoges (Montréal). Le second colloque s'intitulait: Les Pratiques Philosopbiques dans les universités du Québec: perspectives idéologiques, avec comme participants: G. Godin, président et G. Dandenault (Sherbrooke), R. Houde (Montréal), C. Savary (Trois-Rivières), L. Valcke (Sherbrooke), A. Vidricaire (UQAM), P. Laberge (Ottawa).

Le prochain Congrès de l'Acfas aura lieu à l'Université de Moncton, du 7 au 9 mai 1975.

Le $18 \mathrm{e}$ Congrès annuel de l'Association Canadienne de Philosophie avait lieu à l'Université de Toronto, du 30 mai au 3 juin 1974. La collaboration francophone à ce congrès bilingue 
comprenait les communications suivantes: T. De Koninck (Laval), L'intelligence des indivisibles selon Aristote, P. Gravel (Montréal), Hume et l'économie, P. Gagné (Trois-Rivières), La politique de Hobbes, F. Duchesneau (Ottawa), Leibniz et la théorie physiologique, M. Gagnon (Sherbrooke), Une analyse sémantique du concept de causalité est-elle possible?, Y. Gauthier (Montréal), Une théorie de toutes les théories est-elle possible?, R. Champagne (Sudbury), Le thème de la mort dans la pbilosopbie de K. Jaspers, H. P. Cunningham (Laval), Existe-t-il une preuve de la liberté chez Aristote?, J. G. Meunier (UQAM), La Technologie éducative dans l'enseignement de la philosophie: une expérience, G. Legault (Montréal), Meurseault est coupable de meurtre, S. MORIN (Moncton), Pucetti et Cie, G. Bouchard (Laval), Les principales tendances de la sémiologie, $\mathrm{O}$. Reboul (Montréal), Slogan et Enseignement, H. Suligoj (Humber College), La Philosopbie Mystique de A. Watts, J. G. Meunier (UQAM), L'analyse des textes par ordinateurs : le système SATO, C. Murin (Montréal), L'unité de l'intention dans l'oeuvre de Nietzsche, D. Letocha (CEGEP de Rosemont), L'éternel retour chez Niezsche: une faillite pbilosoplique, L. Valcke (Sherbrooke), Le solipsisme cartésien, J. Wojciechowski (Ottawa), Ecologie de la connaissance, J. Theau (Ottawa), Le rapport quantité/qualité chez Hegel et Bergson, Y. Blanchard (Montréal), Le travail comme médiateur de la relation Hegel et Marx, D. McDonell (Ottawa), Le concept de rupture chez Bachelard, Altbusser et Foucault, G. Godin (Laval), Les infinis qui limitent la science, P. Germain (Sir George Williams), Les philosophies de la reconstruction.

Les francophones ont aussi participé à plusieurs sessions bilingues. Une session bilingue, présidée par G. Bouchard (Laval) portait sur les Aspects philosopbiques et linguistiques de la controverse de la linguistique générative et interprétative. Cette session conjointe avec l'Association Canadienne de Linguistique groupait les participants suivants: D. Lightfoot (Linguistics, McGill), Y. Morin (Linguistique, Montréal), T. P. Brodeur (UQAM). Egalement en session bilingue et conjointe avec l'Association Canadienne de Linguistique, un atelier a eu lieu sur Les données et les théories des linguistes avec comme participants: 
W. J. Baker (Linguistics, Alberta), J. Y. Morin (Linguistique, (McGilil) et comme président J. McGilvray (McGill). Une troisième session bilingue sur le thème «Tenses», sous la présidence de J.-P. Brodeur (UQAM) regroupait J. A. McGilvray (McGill), R. Binnick (Linguistics, Toronto) et T.R. Hofmann (Linguistics, Ottawa). Dans une quatrième session bilingue et conjointe avec l'Association Canadienne de Linguistique, session présidée par N. Lacharité (UQAM), J. King-Farlow et $F$. J. Pelletier (Alberta) présentèrent une communication sur le thème La référence chez Zénon. Une session bilingue sur la phénoménologie de Merleau-Ponty, présidée par C. Panaccio (Université du Québec à Trois-Rivières) groupait comme participants: Th. Geraets (Ottawa) et G. B. Madison (McMaster). Un atelier bilingue sur Heidegger et la Métaphysique, présidé par J. Goulet (Sherbrooke) regroupa les participants suivants: E. Joos (Loyola), L. Giroux (Trois-Rivières), P. Gravel (Montréal) et Z. Adamczewski (Brock). Un colloque bilingue eut lieu sur L'enseignement et l'endoctrinement moral, présidé par J. Espinaco-Virseda (Alberta) auquel participèrent $\mathrm{O}$. Reboul (Montréal), M. Schleifer (McGill) et W. H. Neilsen (Manitoba).

Le prochain Congrès de l'ACP aura lieu à Edmonton (Alberta).

Le XVIe Congrès des Sociétés de Philosophie de Langue Française se tenait à Reims (France) du 3 au 6 septembre 1974. A cette occasion la délégation canadienne et québécoise présenta les communications suivantes: A. Klimov (Trois-Rivières), Culture et Anti-Culture chez Nicolas Berdiaeff, M. Renault (TroisRivières), L'ambiguité de la culture entre l'étbique et la civilisation, C. Savary (Trois-Rivières), Sur le concept de culture comme problème philosophique, F. Duchesneau (Ottawa), $M u$ tations intellectuelles et bistoire des sciences, Y. Lafrance (Ottawa), De la nature à la culture selon Platon, Th. Geraets (Ottawa), M. Lagueux (Montréal), Y. Gauthier (Montréal). M. Klimov a également présidé à ce même congrès deux commissions : «La Commission Nicolas Berdiaeff 》 et «La Commission Max Scheler ». M. Yvon Lafrance était le délégué de l'Association Canadienne de Philosophie à ce Congrès et M. V. Cauchy le délégué de la Société de Philosophie du Québec. 


\section{LA SOCIÉTÉ DE PHILOSOPHIE DU QUÉBEC}

Lors de sa première assemblée générale tenue le 9 mai 1974 à l'Université Laval, la Société de Philosophie du Québec a procédé à l'adoption des statuts qui guideront la marche de ses activités et à la nomination des membres de son Bureau de direction et de son Conseil d'administration.

M. Paul-André Quintin (UQTR) a été élu président; Paul Germain (Univ. Sir George Williams) 1er vice-président; Raymond Brouillet (Univ. Laval) 2e vice-président; Olaude Panaccio (Université du Québec à Trois-Rivières) secrétaire ; Yvan Cloutier (CEGEP de Sherbrooke) secrétaire-adjoint; Pierre Gravel (Univ. de Montréal) trésorier.

Outre les membres du Bureau de direction, le Conseil d'administration est composé de représentants des diverses régions du Québec et d'autres lieux de francophonie: pour Chicoutimi, Claude Vallières (CEGEP de Chicoutimi); pour l'Acadie, Mlle Corinne Galant et Maurice Rainville (Univ. de Moncton); pour Montréal, Robert Nadeau (UQAM), Maurice Lagueux (Univ. de Montréal) et Mlle Lise Bouchard (CEGEP MargueriteBourgeois) ; pour Ottawa et l'Outaouais, Pierre Laberge (Univ. d'Ottawa) et Bernard Ouellet (CEGEP de Hull); pour Québec, Guy Bouchard (Univ. Laval) et Maurice Bailly (CEGEP F.-X. Garneau); pour Rimouski, Claudette Lafond (CEGEP de Rimouski) ; pour Sherbrooke, Louis Valcke et Jacques Plamondon (Univ. de Sherbrooke) ; pour Trois-Rivières, Alain Lallier (CECEP de Trois-Rivières) et Claude Savary (UQTR).

A titre de président sortant, M. Venant Cauchy (Univ. de Montréal), président fondateur de la S.P.Q. fait partie du Conseil d'administration pour une période de deux ans.

Pendant cette première année d'existence, la S.P.Q. s'est donnée comme priorité de susciter et supporter les activités régionales et d'assurer l'information à ses membres sur ces activités. Cette priorité s'inscrit par ailleurs dans les buts généraux de la Société qui sont les suivants: 
a) assurer une diffusion aussi complète que possible d'informations concernant la discipline et la profession;

b) faciliter les échanges entre les divers milieux francophones;

c) favoriser les études philosophiques par la discussion, la recherche et les publications;

d) assumer le rôle de porte-parole de la discipline et de la profession auprès des organismes gouvernementaux ou autres.

La société comprend à la fois des membres ordinaires (enseignants ou non-enseignants), des membres associés et des membres institutionnels.

Ie Siège social et le secrétariat administratif de la Société sont à Montréal, 2910 Boulevard Montpetit.

\section{LA SOCIÉTÉ DE PHILOSOPHIE DE MONTRÉAL}

La Société de Philosophie de Montréal a tenu son assemblée généralle annuelle le 30 septembre 1974. Un comité directeur intérimaire a été élu par l'assemblée générale et se compose de MM. Pierre Gravel (Montréal), président, Robert Nadeau (UQAM), vice-président, André Giguère (Collège de Maisonneuve), secrétaire trésorier, Mme Danielle Létocha (Collège de Rosemont), conseillère et $M$. André Mineau, (étudiant, Montréal), conseiller.

Le mandat qui a été confié au comité directeur pour cette année est le suivant:

1. revoir les statuts et prévoir une assemblée générale pour en décider l'adoption;

2. prévoir la publication d'un fascicule bi-semestriel;

3. prévoir et organiser la vie et l'activité philosophiques dans la région de Montréal pour l'année académique 1974-1975. 
Le comité directeur a insisté pour régionaliser la société et en faire un corps intermédiaire qui regrouperait les Universités et les C.E.G.E.P. Des rapports très fructueux ont été ainsi établis entre le département de philosophie de l'Université de Montréal et le département de philosophie de l'Université du Québec à Montréal et les départements de quelques C.E.G.E.P. (Maisonneuve et Rosemont en particulier). Le comité directeur espère obtenir également la participation des Universités anglophones et des contacts sont prévus en ce sens.

Le programme de la société pour cette année a été arrêté dans ses grandes lignes. Les membres seront avertis à l'avance de chaque rencontre. Pour le premier semestre six conférences et un colloque sont prévus. M. Greimas a ouvert les activités de la Société de Philosophie. Suivront les conférences de MM. Vuillemin, Guillermit, Audet, Décarie et Rotta. Le semestre se terminera par un colloque sur Borduas.

\section{ACTIVITÉS DES DÉPARTEMENTS}

\section{a) Université Laval}

M. John R. Gallup et M. Martin Blais ont été promus professeurs titulaires.

M. R. Brouillet a participé au Congrès international sur Kant, à Mayence, Allemagne, en avril 1974; MM. E. Trépanier, W. Murray et L. Ponton au Congrès international de Rome et Naples commémorant le VIIe centenaire de la mort de saint Thomas, du 17 au 24 avril 1974.

La Société brésilienne des philosophes catholiques avait invité l'Université Laval à sa Seconde semaine internationale de philosophie à Petropolis, du 14 au 20 juillet 1974. M. T. De Koninck, délégué de la Faculté, y prononça deux communications intitulées: Es necesaria la filosofia? et Dios y la inteligencia de los indivisibles segun Aristoteles.

MM. G. Bouchard, R.Brouillet, J. Danek, G. Godin et A. Hamel ont participé au Congrès de la Société de philosophie du Québec dans le cadre de l'ACFAS, en mai 1974; MM. G. 
Bouchard, A. Côté, H.-P. Cunningham, T. De Koninck et G. Godin au Congrès de l'Association canadienne de Philosophie, à l'Université de Toronto, en mai-juin 1974.

M. W. Murray a été élu président de la Société internationale d'études aristotéliciennes.

M. R. Brouillet a été élu second vice-président de la Société de philosophie du Québec.

Grâce à la collaboration du Conseil des Arts, M. le professeur Jules Vuillemin, du Collège de France, est l'invité de la Faculté au trimestre d'automne 1974. Du 15 octobre au 14 décembre, il donnera aux étudiants gradués un cours intitulé : «La philosophie de Bertrand Russell et le développement ultérieur de la philosophie analytique».

b) Université de Sherbrooke

Une équipe de chercheurs a entrepris de mettre en chantier une recherche collective qui regroupe sous un seul thème épistémologique des champs d'intérêt multiples. L'intitulé général: Rôle dynamique des antinomies et des paradoxes pour la pensée théorique, caractérise bien un unique souci épistémologique. La recherche examinera tour à tour quatre problématiques: (1) paradoxes et histoire de la philosophie; (2) antinomies logiques et sciences formelles; (3) paradoxes existentiels et communications; (4) sciences de l'homme et dialectique. Les professeurs M. Gagnon, L.-P. Luc, J. Plamondon et J. Tchao participent aux travaux des différents groupes. La coordination des groupes est assurée par un responsable (J. Plamondon) qui voit à promou-voir les échanges entre les différents chercheurs et à maintenir vivant le souci d'intégrer les différents résultats.

Le Centre d'Etudes de la Renaissance organise annuellement un séminaire de recherches interdisciplinaires. Cette année, le séminaire est centré autour de la pensée de Guillaume d'Occam, étudiée à partir de son Expositio in Librum Porpbyrii. Le séminaire est dirigé par M. Louis Valcke (philosophie), avec la collaboration de MM. Martinez de Bujanda (historien, directeur du Centre), Jacques Doyon (théologie), Joseph Tchao (philo- 
sophie). Les travaux de traduction sont assurés par M. Roland Galibois. Dans ce cadre, plusieurs travaux de recherche sont effectués par des étudiants rattachés aux départements de philosophie et d'histoire, ainsi quà la Faculté de théologie.

A la suite de rencontres effectuées dès l'été entre professeurs de philosophie du CEGEP public et de l'Université, ainsi que d'une impulsion venant de la nouvelle Société de philosophie du Québec, on a entrepris de ranimer le Cercle de philosophie, avec objectifs variés et précis, définis dans un projet de constitution, comité général d'organisation, où seront représentés les organismes éducatifs concernés, comités spéciaux selon les besoins, etc. La diversité sera, semble-t-il, de règle: on prévoit des conférences publiques, de la distribution d'information, des ateliers, des rencontres sociales, etc., et cela à différents niveaux de culture (populaire, collégial, universitaire). Autre préoccupation intéressante: créer des liens avec l'activité philosophique du milieu anglophone, c'est-à-dire Champlain College et Bishop's.

\section{c) Université du Québec à Trois-Rivières}

Monsieur le professeur Gilles Lane étant en congé sans solde pour un an, le département de philosophie de l'Université du Québec à Trois-Rivières a accueilli, en septembre, un nouveau professeur, monsieur Claude Panaccio, anciennement du Collège de Maisonneuve. Parmi ses nombreux articles, nous citons: «Conditions et limites de la justification morale», in Pbilosophie et relations interpersonnelles (éd. par A. MONTEFIORE), Montréal, P.U.M., 1973, pp. 147-179; et «Le sujet de la justification morale et le problème de son identité $»$, in Brèches, no 3, hiver-printemps 1974, pp. 41-54.

Dans le cadre du 42e congrès de l'ACFAS tenu à l'Université Laval en mai 1974, plusieurs professeurs du département ont donné des communications: monsieur Paul Gagné, Le problème de la contradiction entre le nationalisme et la lutte des classes; monsieur Marc Renault, De la croyance pré-critique à l'option personnelle; monsieur Claude Savary, A propos d'Edmond de Nevers. 1.-une matrice de production idéologique; 2.-matériaux pour une théorie de l'idéologie, (avec Marc Chabot). 
Par ailleurs, au congrès de l'Association Canadienne de Philosophie tenu à l'Université de Toronto, du $30 \mathrm{mai}$ au 3 juin 1974, monsieur Paul Gagné a présenté la communication suivante La politique de Hobbes et monsieur Laurent Giroux, L'ambivalence de la métaphysique chez Heidegger.

Monsieur Klimov a été nommé directeur de la Collection «Textes et études slaves» publiée aux Presses de l'Université du Québec.

\section{d) Université du Québec à Montréal}

M. le professeur A. Greimas, Ecole Pratique des Hautes Etudes (Paris) a été invité par le département de philosophie à donner quatre séminaires dans le cadre du cours de maîtrise en sémiologie les 24 septembre, 1er, 8 et 15 octobre 1974. Il a de plus prononcé une conférence sur la sémiologie et les sciences humaines, le lundi 30 septembre.

M. le professeur Louis Guillermit de l'Université d'AixMarseille a été invité par le Département de philosophie à prononcer une conférence intitulée «Philosophie et critique» le 16 octobre 1974.

Mme Régine Robin, professeur d'histoire et de linguistique à l'Université de Nanterre a été invitée par M. André Paradis, dans le cadre du cours PHI-411 Idéologies québécoises, à fournir une communication intitulée "Structure du récit dans le reportage de presse: un exemple de presse parisienne en mai 1968».

M. Robert Nadeau, professeur au département de philosophie de I'UQAM, a été élu vice-président de la Société de Philosophie de Montréal, lors de l'assemblée tenue le 30 septembre 1974. Il a également présenté une communication au Congrès sur Kant, 11-14 octobre, organisé par la Faculté de Philosophie de l'Université d'Ottawa.

M. Jean-Guy Meunier, professeur au département de philosophie de l'UQAM, a participé au Congrès international de Linguistique computationnelle tenu à Pise en septembre 1974. M. Meunier continue sa recherche sur un système d'analyse de 
textes par ordinateur, recherche pour laquelle il a obtenu une subvention dans le cadre du plan de Formation de chercheurs et action concertée (FCAC).

Le département de philosophie de I'UQAM a reçu en septembre le manuscrit de la première thèse de maîtrise achevée par l'un de ses finissants. Le manuscrit, intitulé «Proposition d'algorithme pour le dépistage de relations de dépendance contextuelle dans un texte », a été déposé par $\mathrm{M}$. Pierre Plante, qui a fait cette recherche dans l'équipe SATO dirigée par JeanGuy Meunier. M. Plante est chargé de cours à l'Université du Québec à Trois-Rivières durant le semestre d'automne 1974.

e) Université de Moncton

M. Georges François a été nommé directeur du Département de Philosophie. Le Père Louis-Marcel Daigle est en congé sabbatique. M. Maurice Rainville rédige une thèse sur « $\mathrm{La}$ portée ontologique de l'expérience perceptive dans l'oeuvre de Merleau-Ponty ».

M. Serge Morin a donné deux conférences intitulées Two Instances of Structural Injustice et Language as Habitual Activity à I'Université Saint-François-Xavier à Antigonish, les 8 et 9 novembre 1973. Il a donné une conférence sur Le nationalisme et l'injustice de structure à l'Université du Québec à TroisRivières, le 4 décembre 1973.

Mlle Victorine Jabbour a parlé sur L'univers esthétique dans la phénoménologie de Merleau-Ponty au Congrès de l'ACFAS, Université Laval, Québec, mai 1974.

M. Valmond LeBlanc a assisté au début du mois de mai 1974 à la séance «Science et dialectique», au Centre de recherche et de documentation sur Hegel et sur Marx à Poitiers (France).

M. Serge Morin a parlé sur les Désaccords entre les systèmes métaphysiques au Congrès annuel de l'Atlantic Society for Eighteenth Century Studies, à l'Université de Dalhousie à Halifax, mars 1974.

M. Serge Morin a donné les communications suivantes: 
How to Kick the Habit, au troisième congrès annuel de la Société internationale pour l'étude de Husserl et la phénoménologie, Université Sir George William, mars 1974, Je me souviens: critique des théories de Shoemaker, Parfit, Pucetti et al. sur lidentité personnelle, au Congrès de l'ACFAS, Université Laval, Québec, mai 1974, et Roland Pucetti et cie au congrès de l'ACP, Université de Toronto, mai-juin 1974.

M. Maurice Rainville a obtenu une bourse de doctorat du Conseil des Arts du Canada.

M. Valmond LeBlanc a soutenu le 13 mai 1974 sa thèse de $2 \mathrm{e}$ cycle sur La relation dans la logique bégélienne, à l'université de Paris I.

\section{f) Université d'Ottawa}

Le Congrès sur Kant a eu lieu du 10 au 14 octobre 1974. Ce Congrès présidé par $\mathbf{M}$. Pierre Laberge réunit pour quelques jours à Ottawa les grands spécialistes de la philosophie kantienne qui traitèrent des sujets suivants: J.N. Findlay (Boston), Kant Today, J. Vuillemin (Collège de France), Kant Aujourd'hui, G. Funke (Mainz), Die Metaphysische Wende in der KantInterpretation, W.H. Walsh (Edinburg), The Structure of Kant's Antinomies, D.P. Dryer (Toronto), The Second Analogy, A.W. Wood (Cornell), Kant on the Rationality of Morals, G. Lebrun (Aix-Marseille), Réflexions sur la critique de la faculté de juger, O. Reboul (Montréal), Hegel et le formalisme kantien, E. Gerwin(former Councillor for the Federal Republic of Germany to the United Nations), The Idea of a Society of Nations, J.R. Silber (Boston), The Primacy of Practical Reason: a View of the Second Critique, L. Guillermit (Aix-Marseille), De la critique de la raison pratique, J. Taminiaux (Louvain), De la critique de la faculté de juger, G. Schrader Jr. (Yale), Feeling and Form in Kant's Critique of Judgement, T.C. Williams (Guelph), Herder's Essay on Language as the Cameo Model of the Critique of Pure Reason, J.D. McFarland (York), The Bogus Unity of Kantian Philosophy, R.E. Butts (Western Ontario), Kant and the Problem of Scientific Metbodology, J. Robinson (McGill), Hegel's Criticism of the Postulates of Practical Reason, W. Sellars 
(Pittsburgh), Kant's Transcendental Idealism, L. White Beck (Rochester), The Meta-Critique of Pure Reason. Une série de communications brèves ont été données sur les thèmes suivants: Jean Theau (Université d'Ottawa), Critique kantienne et Critique néo-positiviste de la métaphysique, Robert Nadeau (Université du Québec à Montréal), Les Eléments kantiens de la Philosophie du Langage de Ernest Cassirer, Paul-André Quintin (Université du Québec à Trois-Rivières), $Y$ a-t-il lieu de parler d'un Ego Transcendental chez Kant? Question d'un Husserlien, Patricia A. Crawford (San Diego State University), Kand and the Refutation of Scepticism, Allen Buchanan (University of North Carolina), Kant's Second Analogy, Peter Krausser (Freie Universitat Berlin), On some Arguments of Special Deduction in Kant's Chapter on the Analogies, Charles A. Corr (Southern Illinois University, Edwardsville), Analytic and Synthetic Method in Kant, Evan William Cameron (Boston University), A Prescriptive Criterion for Distinguisbing Analytic from Synthetic Judgments, Richard E. Aquila (Duke University), Categories, Schemata and Forms of Judgments, Louise Marcil Lacoste (McGill University), Une Pbilosopbie du sens commun, Claude Buffier (1661-1737) et la Distinction kantienne des Jugements A Priori, Martin G. Kalin (DePaul University), What Makes an Argument Transcendental? A Study in Kant's Logic, PaulEmile Vignola (Université du Québec à Rimouski), Seele et Gemut selon Kant, George Bilek (Ryerson Polytechnical University), What is Pure Reason According to the Critique of Pure Reason, Elenore Vetter-Schrott (University of Vienna/ University of Edinburgh), What Could an Empirical Deduction Be, What a Pbysiological Derivation? A confused Student's Request for Help, Gordon Treash (Mount Allison University), Kant's Only Possible Argument and Critique, Mary-Barbara Zeldin (Hollins College), Kant's Postulate of Immortality, Sidney Axinn (Temple University), Kand and the Moral Antinomy, John Marshall (University of Virgina), Man as an End in Himself, Marc Renault (Université du Québec à Trois-Rivières), Das Prinzip der Selbsterbaltung, Joseph J. Evans (Immaculata College), The Empirical Employment of Pure Reason, Thomas Auxter (University of Florida), The Teleology of Kant's Ectypal World, J. Paul Rosen (Altamont, New York), Permanence and 
Perception: A note on Kant's Second Edition Refutation of Idealism, Rolf George (University of Waterloo), Kant's Theory of Perception, John McArdle (University of Western Ontario), Kant on the Nature of Geometry, Vladimir Zeman (Concordia University). Le professeur Brian E. Morrisey, à titre de Secrétaireexécutif du Congrès, assura l'organisation générale de ce Congrès. Son travail fut fort apprécié de tous les congressistes.

Au IVe Congrès International sur Kant, tenu à Mayence (Allemagne), en avril 1974, M. Pierre Laberge a donné une communication sur: Pbysico-Théologie et omnisuffisance divine chez Kant et M. François Duchesneau a parlé sur : Kant et la physiologie de l'entendement bumain.

MM. Jean Theau et Théodore Geraets ont participé au Congrès sur Hegel qui se tenait à Moscou en août 1974. M. Theau a donné une communication sur: La place du nombre dans la dialectique bégélienne et bamélienne. M. Géraets a donné sa communication sur: Dialectique et Interrogation. Ce $10 \mathrm{e}$ Congrès International était organisé parr l'International Hegel' Gesellschaft.

Le P. Clément Stroick a participé au Congrès International de Rome et Naples commémorant le VIIe centenaire de la mort de Saint Thomas d'Aquin du 17 au 24 avtil 1974. Le P. Stroick a donné une conférence sur: Eine Parise Disputatio von Jabre Dreissig Hunder Funft (1305).

M. François Duchesneau a participé au Congrès de la Société de Philosophie et d'Histoire des Sciences qui avait lieu à Capri, en avril 1974. Il donna une communication sur: Malpighi, Descartes et les problèmes épistémologiques de l'iatromécanisme.

M. François Duchesneau a été nommé co-éditeur de la revue Dialogue par le Conseil d'administration de l'Association Canadienne de Philosophie. Il succède à $\mathrm{M}$. Venant Cauchy (Montréal) qui dirigeait la revue depuis sa fondation il y a dix ans.

La Faculté a accueilli pour l'année 1974-1975, à titre de professeurs invités, les professeurs Kai Nielsen (University of Calgary) et W. H. Dray (Trent University). 
Les professeurs Th. Geraets et Guy Lafrance ont été promus au rang de professeurs titulaires et $M$. Roch Bouchard au rang de professeur agrégé.

Les professeurs L. Moran, J. Wojciechowski, et M. Carignan jouissent de leur congé sabbatique pour l'année scolaire 19741975.

Le Prof. L. Paquet a été nommé secrétaire de la Faculté. Il succède au Prof. Maurice Carignan.

\section{PUBLICATIONS ET INSTRUMENTS DE TRAVAIL}

\section{Cahiers d'Etudes Médiévales}

Les Cahiers d'Etudes Médiévales s'ajoutent aux deux collections déjà publiées par l'Institut d'études médiévales de l'Université de Montréal: les Publications de l'Institut d'études médiévales et les Conférences Albert-le-Grand.

Les Cahiers d'études médiévales veulent atteindre un public diversifié, composé aussi bien de spécialistes, professeurs, chercheurs et étudiants, que de personnes cultivées intéressées à un aspect ou l'autre de l'histoire du moyen âge.

La collection a déjà fait paraître le premier Cahier : Epopées légendes et miracles, en collaboration, Coédition Bellarmin et Vrin, 1974, 168 pages, \$7.25. Quelques Cahiers sont en préparation: En Collaboration, Problèmes d'bistoire et de philosophie des sciences au moyen âge, B. Allard, La théorie du signe chez Guillaume d'Awvergne, S. Lusignan, Préface au "Speculum majus» de Vincent de Beauvais : réfraction et diffraction, $\mathrm{J}$. de Salisbury, Metalogicon. Traduction française, introduction et notes par Guy H. Allard et Jean-Paul Martel. On peut se procurer les Cahiers en s'adressant aux Editions Bellarmin, 8100, Blvd. Saint-Laurent, Montréal H2P 2L9, P. Qué. Canada.

Colloque commémoratif Saint Thomas d'Aquin

Ce colloque organisé par le professeur Jean-Louis Allard de l'Université d'Ottawa tenait ses assises les 6,7 et 8 mars 1974. Les communications ont été publiées dans le numéro de 
mai 1974 de la revue Eglise et Théologie (Université Saint Paul, 223 Main Street, Ottawa K1S 1C4). On trouvera au sommaire les titres suivants: J. L. Allard, Préface, R. Guindon, Ouverture officielle du colloque pour célébrer le 700 e anniversaire de la mort de frère Thomas d'Aquin, A. C. Pegis, After Seven Hundred Years: St. Thomas Aquinas in 1974, L. Ducharme, L'idée de métapbysique dans les écrits du premier enseignement parisien de saint Thomas d'Aquin, C. Stroick, Commentaire: la Métaphysique de saint Thomas, J. Owens, The Primacy of the External in Thomistic Noetics, J. Croteau, Comments on Father Owen's Paper, G. Langevin, De quel secours peut être saint Thomas d'Aquin pour le théologien de notre temps? Th. R. Potvin, Authority in the Church as Participation in the Autority of Christ According to Saint Thomas, A. Gauthier, Comments on Father Potvin's Paper, R. Bellemare, La Somme de Théologie et la lecture de la Bible, P. E. Dion, Réactions à l'exposé du P.R. Bellemare, B. Garceau, Les Etudes Thomistes (1966-1974).

\section{Publications en Pbilosopbie chez HMH, Montréal}

Les Cabiers du Québec constituent un ensemble de neuf collections (Beaux-Arts, Cinéma, Communications, Droit, Ethnologie, Histoire et Documents, Littérature et Documents, Philosophie, Science Politique) publiées par l'éditeur Claude Hurtubise (Hurtubise, $\mathrm{HMH}$, Montréal). Ces collections ont pris la relève des Cahiers de l'Université du Québec et sont dirigées par M. Robert Lahaise, professeur au Département d'Histoire de I'UQAM. La collection de philosophie est dirigée par Jean Paul Brodeur et Georges Leroux, professeurs au Département de Philosophie de l'UQAM. Elle a un double objectif: d'abord publier des travaux qui puiseront dans la philosophie produite au Québec les éléments d'une histoire culturelle et d'une analyse des idéologies susceptibles de contribuer à l'effort contemporain des sciences humaines. De cette tâche, le livre d'Yvan Lamonde constitue un des premiers jalons et la collection voudrait contribuer à en poser plusieurs autres. La collection veut aussi accueillir des travaux actuels où se laisse lire la figure la plus immédiate du travail théorique. L'ouvrage écrit en collaboration Culture et langage correspond à ce second objectif. A l'origine 
de ce projet se trouve un groupe de professeurs de l'UQAM, mais ceux-ci ont choisi d'intégrer leurs travaux à ceux des Cahiers du Québec pour faciliter la pluridisciplinarité et l'ouverture à des collaborateurs venant d'autres milieux. Ces derniers sont invités à soumettre leurs manuscrits à l'adresse des directeurs de la collection (Dépantement de Philosophie, UQAM, B.P. 8888, Montréal 101).

\section{Ouvrages parus dans Les Cabiers de l'Université du Québec}

Georges Leroux (Ed.) Pourquoi la philosopbie? Montréal, 1968 12ème édition, 1971). Textes de Paul Ricoeur, G. St-Pierre, A. Vidricaire, M. Lagueux, F. Leclair, J.G. Meunier, R. Lambert, S. Lusignan, J. Mignolet.

Yvon Lafrance (Ed.)Démocratie, valeurs et savoir, Montréal, 1970. Textes de Y. Lafrance, C. Corbo, G. Leroux, J. Ho-Jou Tchao, L. Valcke.

Ouvrages parus dans les Cabiers du Québec, Hurtubise/HMH Editeur (Collection Philosophie)

Yvan Lamonde, Historiograpbie de la Pbilosopbie au Québec, 1853-1971, Montréal, 1972, 242 p., index, \$4.50. Cahier no .9. Contexte: Yvan Lamonde, Historiograpbie de la philosopbie au Québec. Textes: Louis Adolphe Paquet, Coup d'oeil sur l'bistoire de l'enseignement philosopbique canadien (1917-1918), Arthur Robert, Aspirations du Canada français. Fondement philosopbique (1922), Hermas Bastien, Quelle sera notre philosopbie? (1925), Lucien Beauregard, La part de M. Isaac Stanislas Désaulniers à l'introduction du thomisme au Canada français vers l'époque de la renaissance religieuse de 1840 à 1855 (19411942), X X X, Sur la condition du philosophe (1953), Jacques Brault, Pbilosopbie et religion (1961), Stanley French, Condérations sur l'bistoire et l'esprit de la pbilosophie au Canada français (1964), Jean-Paul Desbiens, Le pbilosopbe et l'actualité nationale (1964), Jacques Brault, Pour une pbilosopbie québécoise (1965), Fernand Dumont, Sur l'enseignement de la pbilosophie (1969), Normand Lacharité, Calliclès parmi nous: 
essai sur les limites de la pédagogie socratique (1971), Chronologie sommaire, Bibliographie chronologique, Index.

COLLAB. Culture et lanpage, Montréal 1973, 286 p., index \$4.95. Cahier no. 11

\section{Première partie: CULTURE ET CRITIQUE}

Georges Leroux, Violence et volonté, Normand Lacharité, Le privilège de l'événement dans les média d'information, Robert Hébert, De l'intolérabilité: remarques sur le vocabulaire de l'échec, Jean-Guy Meunier, L'illusion d'une critique de la culture, Jean-Paul Brodeur, Culture et Saturation, Robert Nadeau, Notes sur l'utilisation du concept de mentalité en bistoire.

\section{Deuxième partie: EPISTEMOLOGIE}

Michel Dufour, Lacan et Althusser: une rencontre et des promesses, Maurice Lagueux, L'usage abusif du rapport science/idéologie, Marcel Rafie, Idéologie et sciences bumaines : la rupture et puis après, Claude Panaccio, La métaphysique et les noms. Index.

\section{À PARAÎTRE}

Raymond Montpetit, Comment parler de la littérature. Sur les théories contemporaines de la critique et leurs fondements philosophiques.

COLLAB. Tâches pour la philosopbie. Textes de L. Brisson, P. Ricoeur, J. King-Farlow, M. Schleifer, D. Latouche, J. Poulain, G.-G. Granger, Y. Lamonde et B. Lacroix, G. Lane, J.-P. Brodeur.

\section{La collection «L'Univers de la Pbilosopbie»}

La collection vient de mettre sous presses son troisième tome : Philosophies de la Cité qui comprendra les études suivantes en philosophie sociale et politique: G. Leroux, Métapbysique et Politique chez Platon, L. Charette, L'bomme: fondement et but de la Cité selon Aristote, Guy-H. Allard, La Cité de Dieu: 
un nouveau Centre du Monde, A. D'Andrea, Le problème du succès politique dans la pensée de Machiavel, M. J. Silverthorne, L'Esprit des Lois de Montesquieu, P. Gagné, La République de Hobbes, G. Lafrance, Jean-Jacques Rousseau et l'idéal républicain, Y. Lafrance, La Philosophie du Droit de Hegel, J. G. Meunier, Marx: d'une philosophie de la Cité à une théorie de la formation sociale, Y. Filippini, Jacques Maritain: éléments d'une politique bumaniste, J. Roy, La Pbilosopbie Politique d'E. Weil.

La collection «L'Univers de la Philosophie » est dirigée par les professeurs Y. Lafrance et J. King-Farlow, assistés d'un comité consultatif. Deux autres ouvrages sont en préparation: Philosophies de l'homme et Philosophies du savoir. On peut se procurer les volumes de la collection en s'adressant aux Editions Bellarmin, 8100, Blvd. Saint-Laurent, Montréal H2P 2L9, P. Qué.

Le Centre de Recherche et de Documentation sur Hegel et sur Marx dirigé par le professeur Jacques D'Hondt qui donna l'an passé à la Faculté de Philosophie d'Ottawa un cours sur le Marxisme annonce les publications suivantes :

1. Hegel et la Pensée Grecque.

2. Hegel et le Siècle des Lumières.

3. La Logique de Marx.

4. Dom Deschamps et sa Métaphysique.

On peut se procurer ces ouvrages en s'adressant au Centre de Recherche et de Documentation sur Hegel et Marx, 8, rue René Descartes, F 86022 Poitiers (France).

Les Presses de l'Université de Montréal ont publié Essai contre le défaitisme politique (1973) 255 p., du professeur Joseph Pestiau (CEGEP de Saint-Laurent).

Les Editions Garneau de Québec ont publié Le Dieu Nouveau (1974), 532 p., par André Dangenais. 


\section{REMERCIEMENTS}

Le comité de rédaction tient à remercier les Doyens et Directeurs des Facultés et Départements de Philosophie d'expression française ainsi que les abonnés d'expression française et d'expression anglaise dont la contribution financière a permis la publication de ce second numéro de la revue. Il remercie également le Conseil des Arts du Canada dont la subvention substantielle a permis de rencontrer les obligations financières de l'impression et de la mise sur le marché du premier numéro de la revue.

Le Directeur: YVON LAFRANCE

\section{WORKSHOP}

Parmenides, Zeno, and their Ancient Critics

The Departement of Philosophy, University of Alberta will, with the support of the Canada Council, host a Research Workshop on the above topic in Edmonton, November 17th-19th, 1974. Copies of research papers will be available beforehand to those attending the Workshop. Results will be summarised at the sessions, to allow maximum time for discussion. There will be no concurrent sessions.

Featured speakers: Richard Bosley (Alberta); David Furley (Princeton) ; Alexander Mourelatos (Texas); T. M. Robinson (Toronto) ; Michael Stokes (Durham); Charlotte Stough (Santa Barbara). Commentators: Brian Calvert (Guelph); Allan Cobb (York); L.J.C. Mix (Alberta); F. J. Pelletier (Alberta); W. M. Pfeiffer (Winnipeg); Roger Shiner (Alberta). Further details from: Roger A. Shiner, Chairman, Workshop Programme Committee, Department of Philosophy, University of Alberta, Edmonton, Alberta, Canada T6G 2E1. 


\section{CANADIAN JOURNAL OF PHILOSOPHY}

September $1974 \quad$ Vol. IV Number 1

Contents: J.-M. Rist, Aristotle: The Value of Man and the Origin of Morality; B. Suits, Aristotle on the Function of Man; S. Munsat, The-Meaning-of-a-Word; B. Szabados, Self Deception; H. Schwyzer, Essence without Universals; S. Malinovich, Knowledge and Evaluation; R. Bradley, The Causal Principle; $M$. Ayers, Individuals without Sortals; P.A. Ostien, God. other Minds, and the Inference to the Best Explanation; L.W. Sumner, Toward a Credible View of Abortion; L.M.G. Clark, Reply to Sumner on Abortion; R.I. Sikora, Rorty's Mark of the Mental and his Disappearance Theory; R. Rorty, More on Incorrigibility; James Ford, Richard Robinson on Incorrigibility; J.F.M. Hunter, Critical Notice of P.M.S. Hacker, Insight and tilusion.

Subscriptions should be sent to: Managing Editor, Department of Philosophy, The University of Alberta, Edmonton, Alberta, Canada T6G 2E1. Institutions: $\$ 12.00$, Individuals: $\$ 10.00$, Students: $\$ 5.00$, Single issues: $\$ 3.00$. 